\title{
Multiple Description Image Coding Using Extended X-tree
}

\author{
Li Li, Canhui Cai, Jing Chen, and Huanqiang Zeng
}

School of Information Science and Engineering, Huaqiao University, Xiamen, 361021, China

Keywords: multiple description coding; dual-tree discrete wavelet transform; $x$-tree image coding.

\begin{abstract}
This paper presents a novel multiple description image coding scheme called extended xtree based multiple description image coding. First, the $2 \mathrm{D}$ dual-tree discrete wavelet transform (DDWT) is exerted on the original image to produce 2 wavelet trees. A noise shaping algorithm is used to reduce the redundancies of the resulted wavelet coefficients produced by DDWT, forming the primary parts of two descriptions. In order to enhance the quality of the side reconstruction, a side noise shaping algorithm is then used to produce the supplementary information of side decoding. The primary information from one tree and supplementary information from the other are grouped to form a description. To exploit the strong correlation between two wavelet trees resulted from DDWT, an extended x-tree coding scheme is used to jointly encode two wavelet trees to improve the coding efficiency. Two descriptions are dispatched through separate channels. Since each description includes critical information of the picture, in case that one of the descriptions is lost, the reconstructed image can still keep acceptable fidelity. Simulation results have demonstrated the superior performance and error resilient ability of the proposed algorithm.
\end{abstract}

\section{Introduction}

Multiple Description Coding (MDC) is a very efficient framework to achieve robust communication over unreliable channels, e.g., lossy packet networks [1]. MDC provide robust transmission over an unreliable communication network with multiple channels by encoding the source into multiple descriptions. Each description contains primary information and some supplementary information of the source. The reconstruction quality depends on the number of descriptions received. When all descriptions are available at the receiver, the decoder can reconstruct the source with the highest fidelity. However, if only a subset of the descriptions is received (signals in one or more channels are lost), the quality of the reconstructed signal should be still acceptable.

Since Vaishampayan presented first MDC scheme, Multiple Description Scalar Quantization (MDSQ) [2], many MDC approaches have been proposed. Some of them are implemented in wavelet domain, such as Polyphase Transform and Selective Quantization (PTSQ) [3], Multiple Description Wavelet Based Image Coding [4], Optimized Multiple Description Lattice Vector Quantization for Wavelet Image Coding [5], Feature-Oriented Multiple Description Wavelet-Based Image Coding [6], to name a few.

Considering that the outcomes of the dual-tree wavelet coefficients consist of two complementary subsets of wavelet coefficients, and each of them can serve as one description, a novel multiple description image coding scheme, called extended $\mathrm{x}$-tree based MDC, is proposed in this paper. First, a 2D dual-tree discrete wavelet transform (DDWT) is applied to the source image to produce 2 sets of wavelet coefficients (i.e., two wavelet trees). Each wavelet tree forms the primary part of a description. To improve the side decoding quality when one description is correctly received, some key coefficients from the other wavelet tree are adopted as the supplementary part of this description. The primary information and supplementary information are grouped to form one description. In order to take the advantage of the strong correlation between two wavelet trees resulted from DDWT to promote the coding performance, an extended x-tree wavelet image coding method [7] is then introduced to encode these two descriptions.

The structure of the remaining parts of this paper is organized as follows: An overview of the DDWT is described in Section 2. Section 3 describes extended $x$-tree image coding algorithm. The 
proposed MDC scheme is introduced in Section 4. Experimental results are provided in Section 5. Conclusions are drawn in Section 6.

\section{Overview of Dual-Tree Image Coding}

It is well known that the traditional critically-sampled discrete wavelet transform (DWT) has two major shortcomings, shift variance and poor directional selectivity. In order to make up the weakness of DWT, Kingsbury proposed a new wavelet transform called dual-tree complex wavelet transform (DT-CWT). A 1-D DT-CWT framework is shown in Fig.1. When the filter pairs, $\left(\mathrm{h}_{0}, \mathrm{~g}_{0}\right)$ and $\left(\mathrm{h}_{1}, \mathrm{~g}_{1}\right)$, approximately obey the Hilbert transform, DT-CWT is nearly shift invariant with higher directional selectivity. This directional sub-band decomposition benefits coding performance for rich textural images.

However, the DT-CWT is an over-complete transform with plenty of redundancy $\left(2^{\mathrm{n}}: 1\right.$ for an $n$ dimensional signal). To reduce this redundancy, Selesnick and Li proposed the dual-tree discrete wavelet transform (DDWT) by using only real part of wavelet coefficients to halve the redundancy of the 2-D DT-CWT but keep its directional selectivity. Fig. 2 shows the directions of the six subbands of 2-D DDWT $\left( \pm 15^{\circ}, \pm 45^{\circ}\right.$ and $\left.\pm 75^{\circ}\right)$.

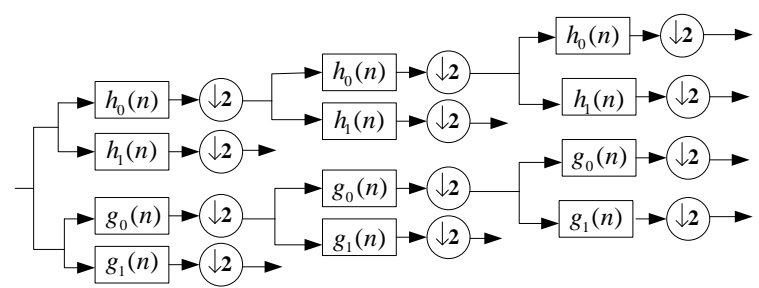

Fig.1: The framework of 1-D DT-CWT

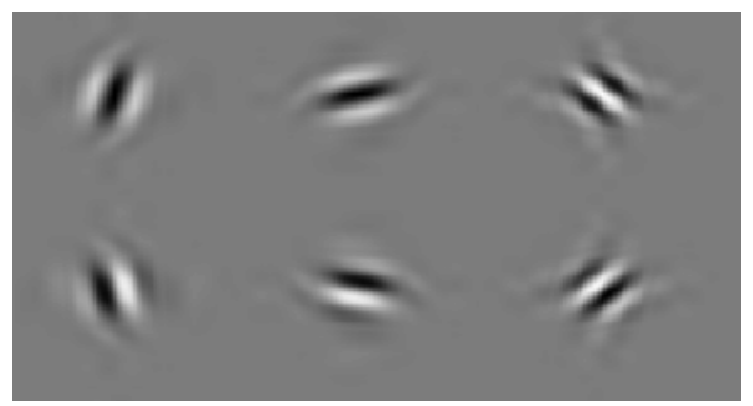

Fig.2: The six directions of 2-D DDWT

Even so, the coefficient number of 2-D DDWT is still twice as that of critically sampled 2-D DWT. Therefore, how to reduce its redundancy is the most concerned for a DDWT-based encoder. Although DDWT uses a large number of wavelet coefficients to represent the original image, it is possible to use much fewer significant coefficients to well approximate the input image. On this light, Reeves and Kingsbury proposed the noise shaping algorithm [8] to deliberately eliminate redundancies. Fig. 3 shows the framework of noise shaping.

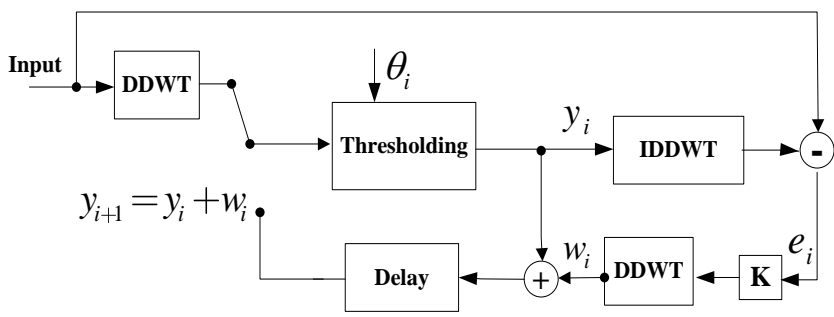

Fig.3: The framework of noise shaping

The noise shaping algorithm iteratively picks up the coefficients with best contribution to the quality of reconstructed image. As a result, the number of significant coefficients is considerably decreased. Actually, the noise shaping is an iterative projection between the image domain and the 
wavelet transform domain. While small coefficients are removed, the significant coefficients are accordingly modified to minimize the error image in spatial domain to compensate the error introduced by the loss of insignificant coefficients.

\section{Extended X-tree Image Coding}

An x-tree is a hierarchical inter-band quad-tree [9], as shown in Fig.4. The offspring of the x-tree must take zero value, but the root of an $\mathrm{x}$-tree can be any real number. A node not belonging to any $\mathrm{X}$-tree is called an isolated node. There are two $\mathrm{x}$-tree coding methods. Since the Stack X-Tree algorithm is much simpler, it is employed to implement our DDWT-based image codec in this paper. The stack $\mathrm{x}$-tree coding scheme separates the information of isolated nodes and $\mathrm{x}$-tree roots into two parts: stacks and an $\mathrm{x}$-tree map. A stack is actually a significant coefficient, and the $\mathrm{x}$-tree map records the position of all $\mathrm{x}$-tree root nodes and isolated nodes, indicating whether a root node is significant or not.

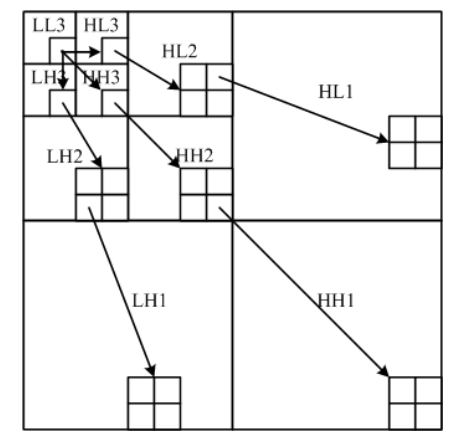

Fig.4: Hierarchical inter-band quad-tree

Since the two wavelet trees produced by DDWT depict the same picture, the locations of $\mathrm{x}$-tree roots in both trees must be highly correlated [7]. Let $\mathrm{N}_{1}(\mathrm{i}, \mathrm{j})$ and $\mathrm{N}_{2}(\mathrm{i}, \mathrm{j})$ denote the coefficients of wavelet tree 1 and wavelet tree 2 at location $(i, j)$, respectively. The similarity of both trees is defined as the percentage of $D_{1}(i, j)=D_{2}(i, j)$, where

$$
\begin{aligned}
& D_{1}(i, j)= \begin{cases}0 & N_{1}(i, j) \in \text { x-tree root } \\
1 & N_{1}(i, j) \in \text { isolate node } \\
2 & N_{1}(i, j) \in \text { descendant node }\end{cases} \\
& D_{2}(i, j)= \begin{cases}0 & N_{2}(i, j) \in \text { x-tree root } \\
1 & N_{2}(i, j) \in \text { isolate node } \\
2 & N_{2}(i, j) \in \text { descendant node }\end{cases}
\end{aligned}
$$

Table. 1 documents the similarity on two standard grayscale $512 \times 512$ images, Lena and Barbara, at several different bit-rates. From this table, one can see that the similarity between the two trees resulted from DDWT are very high. To effectively make use such correlation, we design a dual xtree map to jointly indicate the positions of $x$-tree root of both trees. Each node of the dual $x$-tree map includes two trees' messages and records the state of two trees of DDWT at the same location (i, j). Nine symbols, ZERO, SIG, SZ, ZS, ZROOT, SIGROOT, SZROOT, ZSROOT, and DESC are used to express the dual $x$-tree map, which are defined in Table 2. In this table, $\mathrm{N}_{1}(\mathrm{i}, \mathrm{j})$ and $\mathrm{N}_{2}(\mathrm{i}, \mathrm{j})$ denote the nodes of wavelet tree 1 and wavelet tree 2 at location (i, j).

Table 1: Similarity on "Lena" and "Barbara"

\begin{tabular}{llll}
\hline Rate(bpp) & Lena & Barbara & Rate(bpp) \\
\hline 0.25 & $95.85 \%$ & $93.64 \%$ & 0.25 \\
0.50 & $91.86 \%$ & $90.41 \%$ & 0.50 \\
1.00 & $82.18 \%$ & $85.07 \%$ & 1.00 \\
0.25 & $95.85 \%$ & $93.64 \%$ & 0.25 \\
0.50 & $91.86 \%$ & $90.41 \%$ & 0.50 \\
\hline
\end{tabular}

Table 2: The meaning of symbols for the dual x-tree map

Symbol Meaning




\begin{tabular}{|c|c|}
\hline ZERO & $\begin{array}{l}\text { Both } \mathrm{N}_{1}(\mathrm{i}, \mathrm{j}) \text { and } \mathrm{N}_{2}(\mathrm{i}, \mathrm{j}) \text { are insignificant } \\
\text { nodes. }\end{array}$ \\
\hline SIG & $\begin{array}{l}\text { Both } \mathrm{N}_{1}(\mathrm{i}, \mathrm{j}) \text { and } \mathrm{N}_{2}(\mathrm{i}, \mathrm{j}) \text { are significant } \\
\text { nodes. }\end{array}$ \\
\hline $\mathrm{SZ}$ & $\begin{array}{l}\mathrm{N}_{1}(\mathrm{i}, \mathrm{j}) \text { is significant but } \mathrm{N}_{2}(\mathrm{i}, \mathrm{j}) \text { is } \\
\text { insignificant. }\end{array}$ \\
\hline $\mathrm{ZS}$ & $\begin{array}{l}\mathrm{N}_{1}(\mathrm{i}, \mathrm{j}) \text { is insignificant but } \mathrm{N}_{2}(\mathrm{i}, \mathrm{j}) \text { is } \\
\text { significant. }\end{array}$ \\
\hline ZROOT & Both $\mathrm{N}_{1}(\mathrm{i}, \mathrm{j})$ and $\mathrm{N}_{2}(\mathrm{i}, \mathrm{j})$ are zero roots. \\
\hline SIGRO & $\begin{array}{l}\text { Both } \mathrm{N}_{1}(\mathrm{i}, \mathrm{j}) \text { and } \mathrm{N}_{2}(\mathrm{i}, \mathrm{j}) \text { are significant } \\
\text { roots. }\end{array}$ \\
\hline SZROOT & $\begin{array}{l}N_{1}(i, j) \text { is a significant root but } N_{2}(i, j) \text { is a } \\
\text { zero root. }\end{array}$ \\
\hline ZSROOT & $\begin{array}{l}\mathrm{N}_{1}(\mathrm{i}, \mathrm{j}) \text { is a zero root but } \mathrm{N}_{2}(\mathrm{i}, \mathrm{j}) \text { is a } \\
\text { significant root. }\end{array}$ \\
\hline DESC & $\begin{array}{l}\text { Both } N_{1}(i, j) \text { and } N_{2}(i, j) \text { are descendants of } \\
\text { x-trees. }\end{array}$ \\
\hline
\end{tabular}

\section{The Proposed Multiple Description Coding Algorithm}

Fig.5 shows the framework of proposed dual X-tree MDC system. First, the 2D DDWT is exploited to transform the input image to two wavelet trees, X1 and X2. Second, these two wavelet trees are fed into the central noise shaping algorithm to produce two sparse wavelet trees, X1(R1) and X2(R2) in given bit rates $\mathrm{R} 1$ and $\mathrm{R} 2$. X1 and $\mathrm{X} 2(\mathrm{R} 2), \mathrm{X} 2$ and $\mathrm{X} 1(\mathrm{R} 1)$ are separately input to corresponding side noise shapers. The upper side noise shaping frame produces a noise-shaped wavelet tree X1( $\rho 1)$ according to given bit rate $\rho 1$, and keeps $X 2(R 2)$ unchanged. The $X 1(\rho 1)$ and $X 2(R 2)$ are then combined to form description 1. Similarly, the lower side noise shaping frame produces a noiseshaped wavelet tree $\mathrm{X} 2(\rho 2)$ according to given bit rate $\rho 2$, and keeps X1(R1) unchanged. The $\mathrm{X} 2(\rho 2)$ and $\mathrm{X} 1(\mathrm{R} 1)$ are then combined to form description 2. In this way, we can get the basic information of description from the central noise shaping and the supplementary information from the side noise shaping. Each description includes the basic information and supplementary information. To effectively encode the DDWT coefficients and make full use of the correlation between two wavelet trees of each description, we employ an extended x-tree coder to encode these two descriptions. Finally, two descriptions are dispatched from separate channels.

From Fig.5, one can see that X2(R2) and X1(R1) are employed as the primary parts of the description 1 and description 2, respectively; X1( $\rho 1)$ and $X 2(\rho 2)$ are used as the supplementary parts of the description 1 and description 2, respectively. It's easy to see that the supplementary part of one description is the redundancy of the basic information of another description. When all the descriptions are available at the receiver, X1(R1) and X2(R2) are applied to reconstruct the source image with high fidelity. If only one description is received, for instance, only description 1 is available, then X1( $\rho 1)$ and X2(R2) are used to reconstruct the source image with acceptable quality.

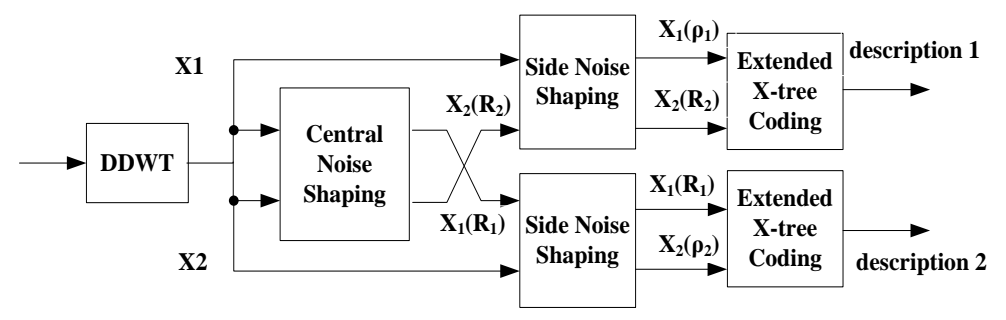

Fig.5: The framework of noise shaping

\section{Experimental Results and Discussion}

To verify the performance of the proposed extended x-tree based MDC algorithm, two standard 8bit grayscale $512 \times 512$ images with multidirectional textures, "Barbara" and "Lena", are used as the testing images to check the performance of the proposed algorithm. Fig.6 and Fig.7 illustrate the 
rate-distortion performance of central/side decoding between our proposed method and that of references [4-6] on the testing images at the same bit rate.

From Fig.6 and Fig.7, one can see that the proposed MDC scheme obviously outperforms the schemes proposed in [4-6]. Fig.6 and Fig.7 also show that quality improvement on "Barbara" is even higher than that on "Lena", because the texture of "Barbara" is with multiple directions which requires the directional selectivity ability of an encoder. Accordingly, the proposed dual xtree MDC performs better at low bit rate situations.

\section{Conclusions}

A new MDC system using dual-tree wavelet transform and extended $\mathrm{x}$-tree is proposed in this paper. First, 2D DDWT is exploited to transform the original image to two wavelet trees. A central noise shaping algorithm is employed to select the wavelet coefficients to form the basic parts of two descriptions and a side noise shaping algorithm is used to draw the supplementary information to improve the quality of side reconstruction. An extended $\mathrm{x}$-tree encoder is applied to jointly encode two wavelet trees produced from DDWT to exploit the correlation between these two trees. Consequently, the coding performance is improved. Simulation results have proved the proposed MDC algorithm outperforms most state-of-the-art image MDC algorithms.

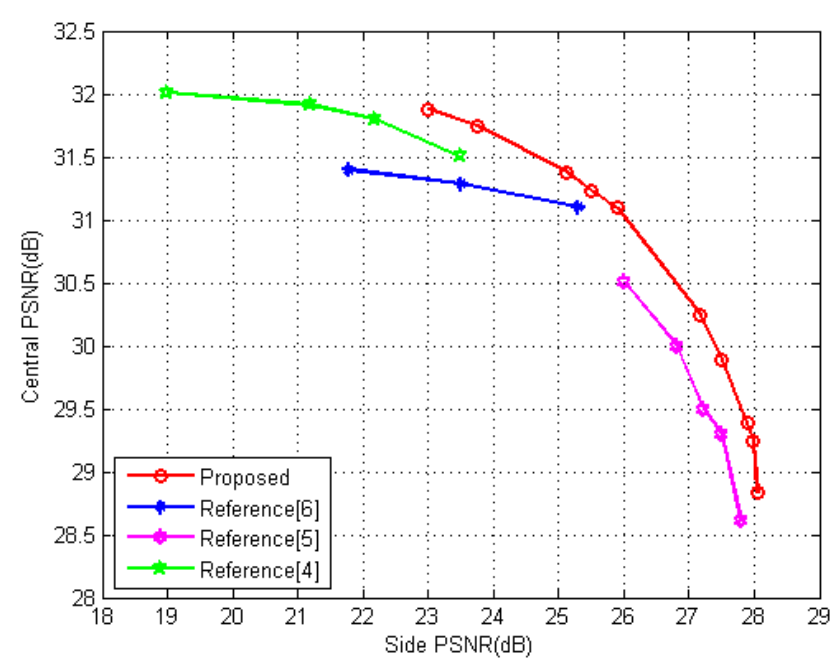

Fig.6: The R-D performance on "Barbara"( $\mathrm{R}=0.5 \mathrm{bpp})$

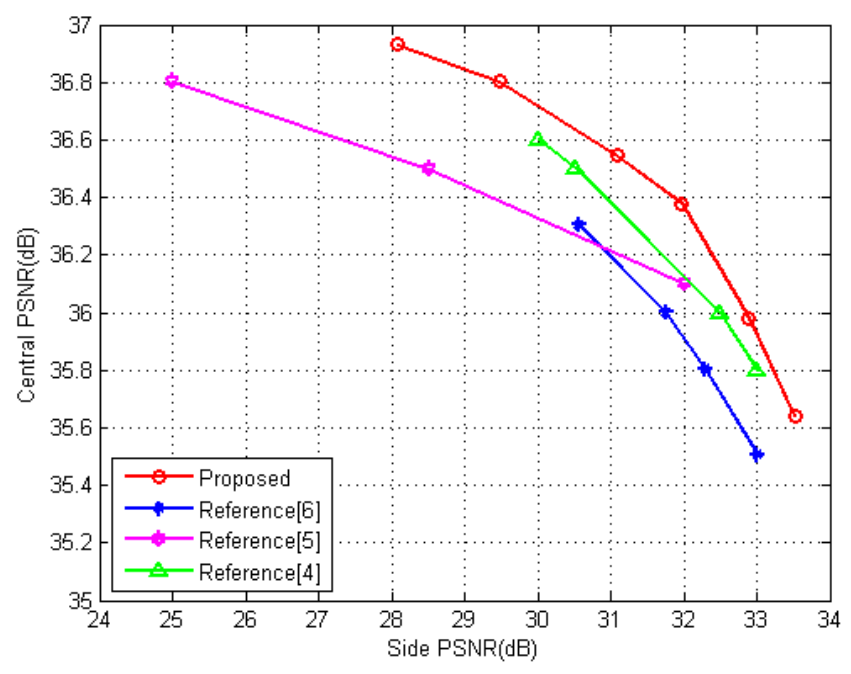

Fig.7: The R-D performance on "Lena" ( $\mathrm{R}=0.5 \mathrm{bpp})$ 
This work was supported in part by the National Natural Science Foundation of China under the Grants 61372107 and 61401167, in part by the Xiamen Key Science and Technology Project Foundation under the Grant 3502Z20133024, and in part by the High-Level Talent Project Foundation of Huaqiao University under the Grants 14BS201 and 14BS204.

\section{References}

[1] Vaishampayan V. A., Design of multiple description scalar quantizer. IEEE Transactions on Inform Theory, 39(5), pp.821-834, 1993.

[2] Jiang W. \& Ortega A., Multiple description coding via polyphase transform and selective quantization. Proceedings of Visual Communications and Image Processing (VCIP), San Jose, CA, USA, pp.998 - 1008, 1999.

[3] Akhtarkavan E., Mohd. Fadzli \& Mohd Salleh, Multiple Descriptions Coinciding Lattice Vector Quantizer for Wavelet Image Coding. IEEE Transactions on Image Processing, 21(2) , pp.653661, 2012.

[4] Bai H, Zhu Ce \& Zhao Y, Optimized Multiple Description Lattice Vector Quantization for Wavelet Image Coding. IEEE Transaction on Circuits and Systems for Video Technology, 17(7) , pp.912-917, 2007

[5] Liu Y \& Oraintara S., Feature-Oriented Multiple Description Wavelet-Based Image Coding. IEEE Transactions on Image Processing, 16(1), pp.121-131, 2007.

[6] Selesnick I. W., Baraniuk R. G. \& N. G. Kingsbury, The dual-tree complex wavelet transform. IEEE Signal Process. Mag., 22(6), pp: 123 - 151, 2005.

[7] Li L. \& Cai C., Dual x-tree Wavelet Image Coding. Proc. International Conference on Signal Processing (ICSP), Beijing, China, pp.716-719, 2010.

[8] Reeves T. H. \& Kingsbury N. G., Overcomplete image coding using iterative projection-based noise shaping. Proceeding of IEEE International Conference on Image Proceeding (ICIP), Rochester, NY, USA, pp.597-600, 2002.

[9] Cai C., Mitra S.K. \& Ding R., Smart wavelet image coding: x-tree approach. Signal Processing, 82(2), pp. 239-249, 2002. 